\title{
Multicaloric materials and effects
}

Enric Stern-Taulats, Teresa Castán, Lluís Mañosa*, Antoni Planes,

Neil D. Mathur, and Xavier Moya*

Multicaloric materials show thermal changes that can be driven simultaneously or sequentially by more than one type of external field, and the resulting multicaloric effects can be large in multiferroic materials. The use of more than one driving field can permit access to larger thermal changes, with smaller field magnitudes, over wider ranges of operating temperature, while manipulating hysteresis. The thermodynamics behind multicaloric effects is well established, but only a small number of multicaloric effects have been experimentally studied to date. Here we describe the fundamentals of multicaloric effects, and discuss the performance of representative multicaloric materials. Exploiting multicaloric effects could aid the future development of cooling devices, where key challenges include energy efficiency and operating temperature span.

Keywords:caloric effects, multiferroic materials, multicaloric materials.

\section{Introduction}

Caloric materials exciting current interest show reversible thermal changes in response to field-induced changes of a ferroic order parameter. Specifically, one may achieve magnetocaloric ${ }^{1}$, electrocaloric ${ }^{2}$ and mechanocaloric ${ }^{3,4}$ effects using magnetic fields, electric fields and stress fields, respectively. In practice, caloric responses are quantified in two thermal extremes, as either the isothermal entropy change or the adiabatic temperature change ${ }^{5-7}$. In recent years, interest has focussed on the development of materials that display large caloric effects near room temperature, for application in solid-state refrigeration devices ${ }^{8}$.

Giant caloric responses are expected to occur in the vicinity of phase transitions, where the properties of the materials are strongly temperature dependent ${ }^{5,6,9}$. Caloric effects that involve latent heat are particularly large, such that first-order ferromagnetic, ferroelectric and ferroelastic materials ${ }^{6,7}$ are particularly promising.

Multiferroic materials are characterized by two or more ferroic properties that may or may not be strongly coupled ${ }^{10}$, thus permitting a given ferroic order parameter to respond to more than one type of applied field. Consequently, different ferroic properties can emerge at nearby temperatures, or even simultaneously. Large caloric effects can thus be driven by the simultaneous or 
sequential applications of more than one type of external field, in what are thus known as multicaloric materials ${ }^{7}$.

The main limitation of materials displaying caloric effects near first-order phase transitions is thermal and field hysteresis ${ }^{11}$. Hysteresis typically arises as a consequence of nucleation, but it instead arise primarily due to pinning associated with the elastic changes that arise in many of the transitions of interest. It was recently shown that hysteresis can be bypassed by exploiting the response of multicaloric materials to more than one type of driving field ${ }^{12}$, although extra work is done when varying the secondary field.

Most giant magnetocaloric and electrocaloric materials (undergoing first-order phase transitions) are expected to also show mechanocaloric effects, as the ferroic order parameter is likely to be strongly coupled to the lattice (Figure 1). Over the last few years, there has been growing interest in studying caloric effects while varying the stress field along with the magnetic or electric field (Refs [27-29], and references therein). So far, most of this type of work has employed hydrostatic pressure as the primary control parameter. By contrast, there has been much less research on multicaloric effects that employ magnetic and electric fields, in spite of the ongoing interest in multiferroic materials and the magnetoelectric effects that they display. In the present paper, we will discuss the state-of-art research on multicaloric materials and multicaloric effects, and we will discuss the prospects for future advances.

\section{Thermodynamics of Multicaloric Effects}

In this section, we will briefly recall the main thermodynamic relations that are required to understand multicaloric responses in materials that display more than one ferroic property. Let us assume the likely scenario of two external fields $Y_{1}$ and $Y_{2}$, which are associated with their two conjugate ferroic variables $X_{1}$ and $X_{2}$, respectively. For example, a magnetic field $\mu_{0} H$ and an electric field $E$ would be associated with sample magnetization $M$ and polarization $P$, respectively. Let us also restrict our discussion to isothermal changes of entropy $\Delta S$, and the assumption of equilibrium thermodynamics.

Given that entropy is a state function, it will undergo path-independent changes that do not depend on whether the driving fields are applied simultaneously, or in some sequence. We may therefore express the entropy change arising due to a change in both applied fields by following any specific path ${ }^{30}$, e.g.:

$$
\Delta S\left[(0,0) \rightarrow\left(Y_{1}, Y_{2}\right)\right]=\Delta S\left[(0,0) \rightarrow\left(Y_{1}, 0\right)\right]+\Delta S\left[\left(Y_{1}, 0\right) \rightarrow\left(Y_{1}, Y_{2}\right)\right] .
$$

Here, the first term on the right is the monocaloric effect due to the application of field $Y_{1}$ at $Y_{2}=0$, while the second term on the right is the monocaloric effect due 
to the application of field $Y_{2}$ at constant $Y_{1}$. One may then express the two terms via the appropriate Maxwell relations to obtain:

$$
\Delta S\left[(0,0) \rightarrow\left(Y_{1}, Y_{2}\right)\right]=\int_{0}^{Y_{1}}\left(\frac{\partial X_{1}}{\partial T}\right)_{Y_{1}^{\prime}, Y_{2}=0} \mathrm{~d} Y_{1}^{\prime}+\int_{0}^{Y_{2}}\left(\frac{\partial X_{2}}{\partial T}\right)_{Y_{1}, Y_{2}^{\prime}} \mathrm{d} Y_{2}^{\prime},
$$

As shown in refs [30, 31], one may expand the second term on the right of Equation 2, such that the expression becomes:

$$
\begin{aligned}
\Delta S\left[(0,0) \rightarrow\left(Y_{1}, Y_{2}\right)\right] & =\int_{0}^{Y_{1}}\left(\frac{\partial X_{1}}{\partial T}\right)_{Y_{1}^{\prime}, Y_{2}=0} \mathrm{~d} Y_{1}^{\prime}+\int_{0}^{Y_{2}}\left(\frac{\partial X_{2}}{\partial T}\right)_{Y_{1}=0, Y_{2}^{\prime}} \mathrm{d} Y_{2}^{\prime} \\
& +\int_{0}^{Y_{1}} \int_{0}^{Y_{2}} \frac{\partial \chi_{12}}{\partial T} \mathrm{~d} Y_{1}^{\prime} \mathrm{d} Y_{2}^{\prime} .
\end{aligned}
$$

In contrast with Equation 2, the entropy change is now expressed as the sum of two monocaloric responses that are determined while the inactive field is zero, and a cross-coupling term that is significant if the cross-susceptibility $\chi_{12}=\left(\frac{\partial X_{1}}{\partial Y_{2}}\right)_{T, Y_{1}}=\left(\frac{\partial X_{2}}{\partial Y_{1}}\right)_{T, Y_{2}}$ exhibits a notable temperature dependence. (This cross-susceptibility represents the magnetoelectric coupling coefficient ${ }^{10}$ when applying magnetic and electric fields.)

Equation 3 shows that the multicaloric response of a material is not given by the sum of each monocaloric response while the inactive field is zero, unless $\partial \chi_{12} / \partial T=0$. This may be appreciated by considering the diagram in Figure 2, where the field variables are magnetic field and pressure. First, it is necessary to observe that path independence implies that one may achieve the same entropy change $(A \rightarrow D)$ either by applying pressure at zero magnetic field $(A \rightarrow C)$ and then magnetic field at finite pressure $(C \rightarrow D)$, or by applying magnetic field at zero pressure $(\mathrm{A} \rightarrow \mathrm{B})$ and then pressure at finite magnetic field $(\mathrm{B} \rightarrow \mathrm{D})$. It then follows that the magnitude of a caloric effect driven by one field variable depends on the magnitude of the other field variable, due to the cross-coupling. For example, the magnetocaloric effects are different in magnitude when driven at zero pressure $(\mathrm{A} \rightarrow \mathrm{B})$ or finite pressure $(\mathrm{C} \rightarrow \mathrm{D})$.

\section{Multicaloric examples}

The ability to vary more than one field gives access to a wider range of parameter space, such that a given phase transition may become accessible with lower fields in a broader window of temperatures. This may be appreciated by considering the (anhysteretic) phase diagrams in Figure $3(a, b)$, where the field variables are again magnetic field and pressure. For example, the $\alpha \rightarrow \beta$ transition in Figure 3(a) can only be induced at finite pressures in the constant-temperature plane that lies at the highest temperatures shown. More generally, the signs of both field changes should be chosen in order to drive the transition in the same direction. For 
example, the $\alpha \rightarrow \beta$ transition in Figure 3(b) is favoured by increasing magnetic field and reducing pressure.

The hysteresis that is in practice associated with first-order phase transitions can be manipulated by judiciously using a secondary applied field. This strategy can shift hysteresis from the primary field to the secondary field ${ }^{12}$, or fully undrive caloric effects that cannot be fully undriven by the removal of the primary field alone. An example of the former scenario is illustrated in Figure 4 and described in the caption, again using magnetic field and pressure as the field variables. The energy dissipated during the multicaloric cycle shown (red dashed line) is given to a good approximation by $|\Delta p \Delta v|$, where $\Delta p=p_{2}-p_{1}$ and $\Delta v$ is the difference in specific volumes for the two phases (Figure 4b) ${ }^{23}$. Experimentally, these kind of multicaloric cycles have been realized using $\mathrm{Fe}-\mathrm{Rh}^{23}$ and $\mathrm{La}-\mathrm{Fe}-\mathrm{Mn}-\mathrm{Si}^{32}$.

The pressure and magnetic field variables were chosen in Figures 2 to 4 because most multicaloric studies focus on magnetocaloric effects that are modified by hydrostatic pressure ${ }^{33-37}$. For example, the peak values of magnetic entropy change in Mn-Ni-Si-based compounds ${ }^{36}$ and Mn-rich Ni-Mn-Ga Heusler alloys ${ }^{37}$ is increased by hydrostatic pressure, whereas there is a decrease in the peak magnetocaloric effect in $\mathrm{Gd}-\mathrm{Si}-\mathrm{Ge}^{34}$ and $\mathrm{Ni}-\mathrm{Mn}-\mathrm{Co}-\mathrm{Sb}$ Heusler alloys ${ }^{38}$. There are also reports on elastocaloric effects that are modified by either magnetic or electric fields [24, 39]. In Heusler alloys, the elastocaloric refrigerant capacity can be increased by $\sim 20 \%$ using magnetic fields of $\mu_{0} H=1 \mathrm{~T}^{24}$. By contrast, the elastocaloric properties of single-crystal ferroelectric oxides are hardly affected by an electric field ${ }^{39}$.

There are still very few reports on caloric effects driven by both magnetic and electric fields. However, it should be possible to electrically control the magnetocaloric effect in multiferroic heterostructures via strain ${ }^{18,40-42}$. For, example a small multicaloric response has been demonstrated using ribbons of the Heusler material Ni-Co-Mn-In on a ferroelectric substrate of PMN-PT $\left[(1-x) \mathrm{Pb}\left(\mathrm{Mg}_{1 / 3} \mathrm{Nb}_{2 / 3}\right) \mathrm{O}_{3}-x \mathrm{PbTiO}_{3}\right]^{18}$, where an electric field modified the peak temperature of the inverse magnetocaloric effects. Similar results were also obtained ${ }^{42}$ in FeRh-films on $\mathrm{BaTiO}_{3}$ substrates.

\section{Outlook}

The use of more than one field variable opens up what may be considered to be a new field of research, just as the use of both magnetic and electric fields has opened up the field of magnetoelectrics with respect to large separate bodies of work on ferromagnetic and ferroelectric materials. The study of multicaloric materials and effects is now particularly timely given the confluence of research in magnetocaloric, electrocaloric and mechanocaloric effects. This confluence is manifested in both review articles ${ }^{5-7,43}$ and international meetings, such as MRS Spring 2016, MRS Spring 2017 and MRS Fall 2018. In addition to a cross-fertilization of expertise, one can anticipate a blossoming of multicaloric 
research that will arise from the overlap, yielding both novel science and possible applications. Consequently, we can say with confidence that the field of multicalorics will continue to be greater than the sum of the parts.

\section{Acknowledgements}

This work was supported by the Spanish Ministry of Science project MAT2016-75823-R, the EPSRC Grant No. EP/P031412/1, and the ERC Starting Grant No. 680032. X. M. is grateful for support from the Royal Society.

\section{References}

1. V. K. Pecharsky and K. A. Gschneidner, Jr, Phys. Rev. Lett. 78, 4494-4497 (1997).

2. B. Neese et al., Science 321, 821 (2008).

3. E. Bonnot, R. Romero, L. Mañosa, E. Vives, and A. Planes, Phys. Rev. Lett. 100, 125901 (2008).

4. L. Mañosa et al., Nature Mater. 9, 478 (2010).

5. S. Fähler et al., Adv. Eng. Mater., 14, 10 (2012), and references therein.

6. L. Mañosa, A. Planes, and M. Acet, J. Mater. Chem. A 1, 4925 (2013), and references therein.

7. X. Moya, S. Kar-Narayan, and N.D. Mathur, Nature Mater. 13, 439 (2014), and references therein.

8. A. Kitanovski, U. Plaznic, U. Tomc, and A, Poredoš, Int. J. Refrig. 57, 288 (2015).

9. A. Planes, L. Mañosa, and M. Acet, J. Phys. Condens. Matter, 21 (2009) 233201 (29 pp).

10. W. Eerenstein, N. D. Mathur, and J. F. Scott, Nature 442, 759 (2006).

11. O. Gutfleisch et al., Philos. Trans. Roy. Soc. 374, 20150308 (2016).

12. J. Liu, T. Gottschall, K.P. Skokov, J.D. Moore, and O. Gutfleisch, Nature

Mater. 11, 620 (2012).

13. P. Lloveras et al., Nature Commun.6, 8801 (2015).

14. S. Crossley, W. Li, X. Moya, and N. Mathur, Philos. Trans. R. Soc. A 374, 20150313 (2016).

15. X. Moya et al., Adv. Mater. 25, 1360 (2013)

16. E. Stern-Taulats et al., APL Mater. 4, 091102 (2016)

17. Y. Yoshida, K. Yuse, D. Guyomar, J. F. Capsal, and G. Sebald, Appl. Phys. Lett. 108, 242904 (2016).

18. Y. Y. Cong et al., Adv. Mater. 27, 801 (2015).

19. J. Tušek, K. Engelbrecht, L. P. Mikkelsen, and N. Pryds, J. Appl. Phys. 117, 124901 (2015).

20. D. Matsunami, A. Fujita, K. Takenaka, and M. Kano. Nat. Mater. 14, 73-78 (2015).

21. J. M. Bermúdez, M. Sánchez-Andújar, S. Castro-García, J. López-Beceiro, R. Artiaga, and M. A. Señarís-Rodríguez, Nat. Commun. 8, 15715 (2017) 
22. E. Stern-Taulats et al., Phys. Rev. B 89, 214105 (2014)

23. E. Stern-Taulats et al., Phys. Rev. B 95, 104424 (2017).

24. P. Castillo-Villa et al., Phys. Rev. B 83, 174109 (2011).

25. S. Yüce et al., Appl. Phys. Lett. 101, 071906 (2012).

26. S. Yu. Dan'kov, A. M. Tishin, V. K. Pecharsky, and K. A. Gschneidner, Phys. Rev. B 57, 3478-3490 (1998).

27. A. Planes, T. Castán, and A. Saxena, Philos. Trans Roy. Soc. 374, 20150304 (2016).

28. L. Mañosa and A. Planes, Adv. Mater. 29, 1603607 (2017).

29. Y. Liu, J. F. Scott, and B. Dkhil, APL Mater. 4, 064109 (2016).

30. A. Planes, T. Castán, A. Saxena, Philos. Mag. 94, 1893 (2014).

31. E. Stern-Taulats, PhD thesis, University of Barcelona (2017), available at http://diposit.ub.edu/dspace/handle/2445/111605

32. E. Lovell et al., Phys. Status Solidi RRL 11, 1700143 (2017).

33. S. Gama et al., Phys. Rev. Lett. 93, 237202 (2004).

34. A. Magnus et al., J. Appl. Phys. 97, 10M320 (2005).

35. J. Lyubina, K. Nenkov, L. Schultz, and O. Gutfleisch, Phys. Rev. Lett. 101, 177203 (2008).

36. T. Samanta et al., Phys. Rev. B, 91, 020401(R) (2015).

37. F. Albertini, J. Kamarád, Z. Arnold, L. Pareti, E. Villa, and L. Righi, J. Magn. Magn. Mater. 316, 364-367 (2007).

38. A. K. Nayak, K.G. Suresh, A.K. Nigam, A.A. Coelho, and S. Gama, J. Appl. Phys. 106, 053901 (2009).

39. A. Chauhan, S. Patel, and R. Vaish, Acta Mater. 89, 384 (2015).

40. X. Moya et al., Nature Mater. 12, 52 (2013).

41. R.O. Cherifi et al., Nature Mater. 13, 345 (2014).

42. Y. Liu et al., Nature Comm. 7, 11614 (2016).

43. S. Crossley, N. D. Mathur, and X. Moya, AIP Adv. 5, 067153 (2015) 


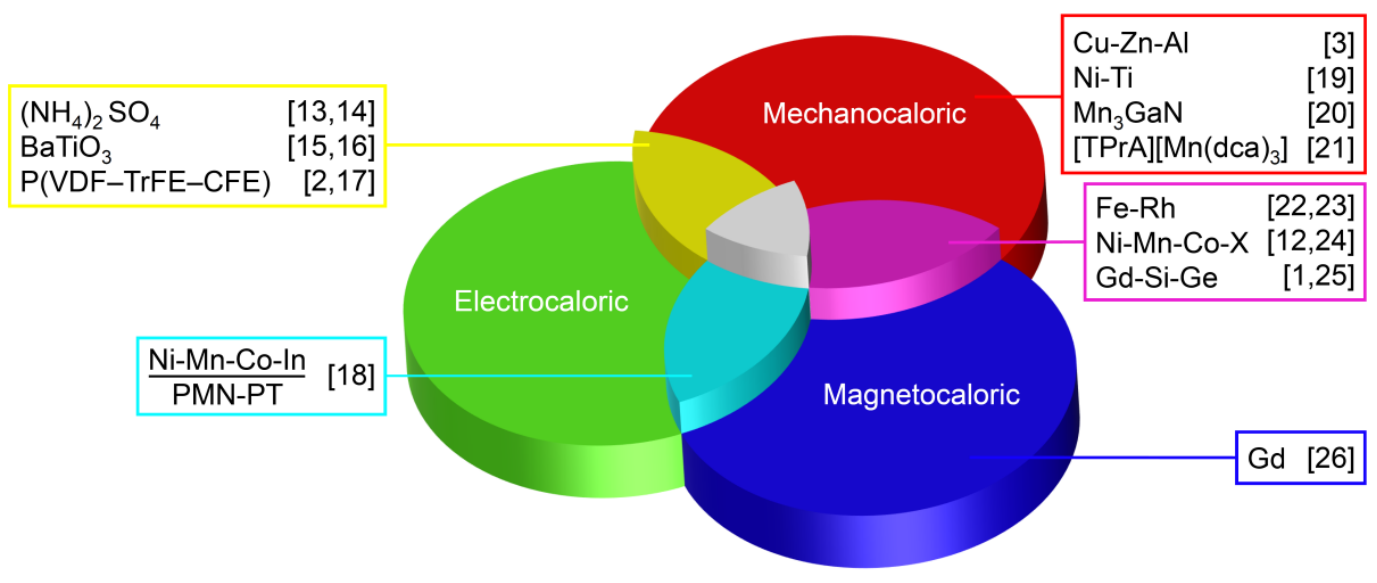

Figure 1. The relationship between magnetocaloric, electrocaloric and mechanocaloric materials. Materials lying at the intersections are multicaloric. Selected examples are based on existing measurements of large caloric effects near room temperature. It is likely that all magnetocaloric and electrocaloric materials will show mechanocaloric effects that may or may not be large. 


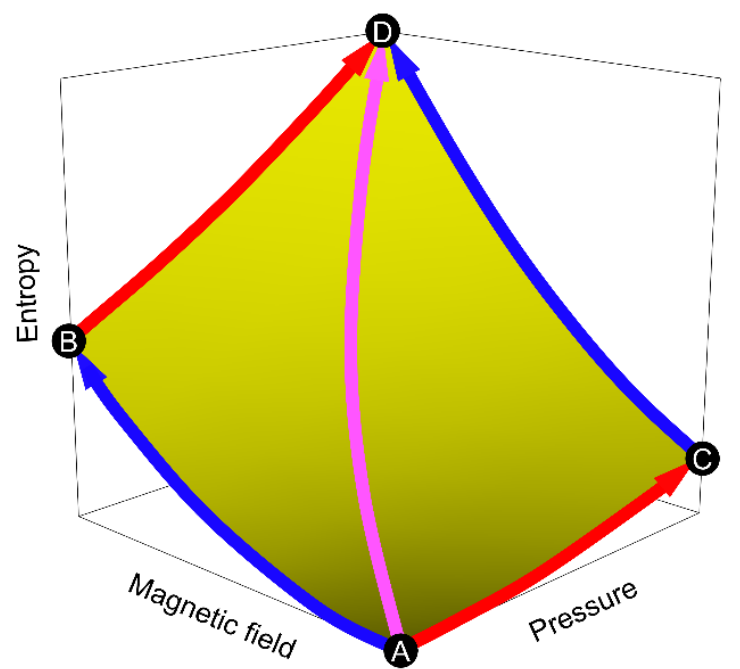

Figure 2.Schematic variation of isothermal entropy with pressure and magnetic field $\left(\chi_{12} \neq 0\right)$. Multicaloric entropy changes can be achieved by applying pressure and magnetic field either simultaneously or sequentially. For example, the entropy change associated with pathway $\mathrm{A} \rightarrow \mathrm{D}$ can be achieved in either one step, two steps [by applying first pressure $(\mathrm{A} \rightarrow \mathrm{C})$ and then field $(\mathrm{C} \rightarrow \mathrm{D})$, or by applying first field $(\mathrm{A} \rightarrow \mathrm{B})$ and then pressure $(\mathrm{B} \rightarrow \mathrm{D})$ ], or multiple steps. 

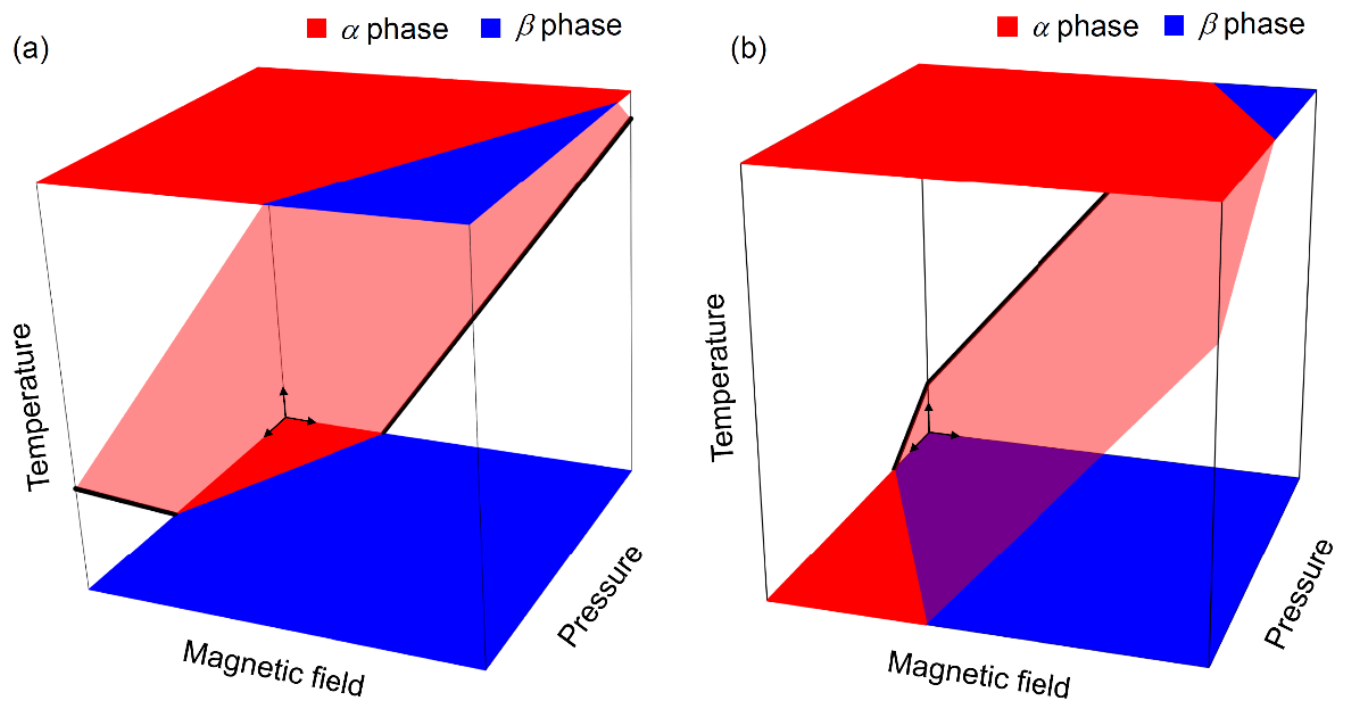

Figure 3. Schematic phase diagrams for materials at finite temperatures, in which transitions between a high-temperature $\alpha$ phase and a low-temperature $\beta$ phase are associated with (a) conventional magnetocaloric effects $(\mathrm{d} T / \mathrm{d} H>0)$ and conventional barocaloric effects $(\mathrm{d} T / \mathrm{d} p>0)$, and (b) conventional magnetocaloric effects $(\mathrm{d} T / \mathrm{d} H>0)$ and inverse barocaloric effects $(\mathrm{d} T / \mathrm{d} p<0)$. Sloping pink planes denote the (anhysteretic) phase boundary. 


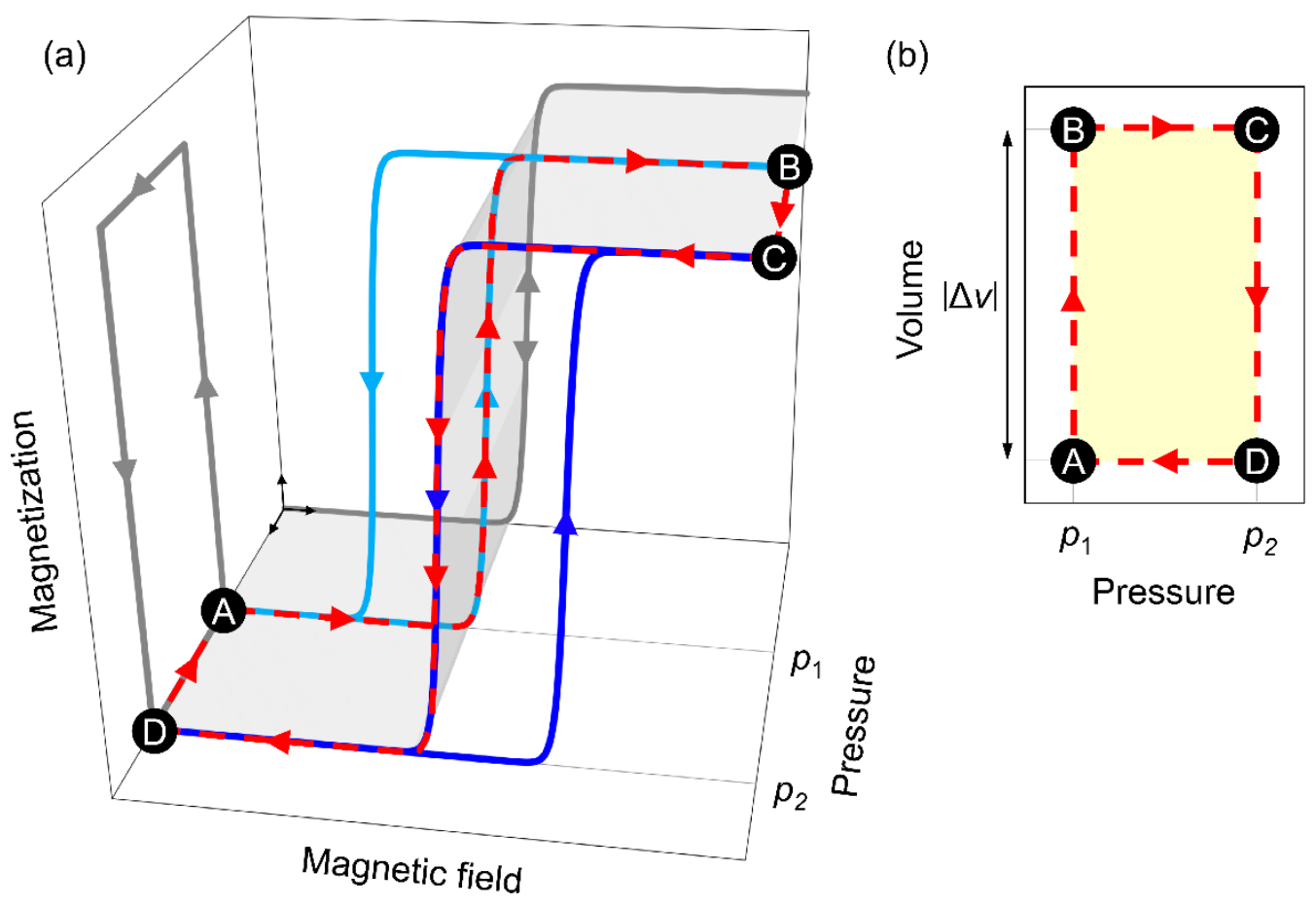

Figure 4. Multicaloric trajectories. (a) The red dashed line $(A \rightarrow D)$ includes isobaric magnetization-field legs at pressures $p_{1}$ (light blue dashed and solid lines) and $p_{2}$ (dark blue dashed and solid lines). Projections of this trajectory along the pressure and field axes (grey solid lines, located for convenience at plot edge) help understand the hysteresis. The projection along the pressure axis shows that magnetic hysteresis can be traded for pressure hysteresis by increasing the pressure before removing the magnetic field. This dissipation is seen by plotting (b) the corresponding changes of volume versus pressure. For simplicity, we assume that the magnetization and volume do not change when the pressure is $\operatorname{varied}(\mathrm{B} \rightarrow \mathrm{C}$ and $\mathrm{D} \rightarrow \mathrm{A})$. 